\title{
Epidemiological Aspects of Recruitment of Male Volunteers for Non-Invasive Urodynamics
}

\author{
V. Avagyan ${ }^{a} \quad$ R. van Mastrigt ${ }^{a} \quad$ J.W.N.C. Huang Foen Chung ${ }^{a} \quad$ A.M. Bohnen ${ }^{c}$ \\ J.L.H.R. Bosch ${ }^{a}$ P.G.H. Mulder ${ }^{b}$
}

Departments of ${ }^{\mathrm{a}}$ Urology, Sector Furore, ${ }^{\mathrm{b}}$ Epidemiology and Biostatistics, and ${ }^{\mathrm{c}}$ General Practice, Erasmus MC, Rotterdam, The Netherlands

\section{Key Words}

Benign prostatic hyperplasia - Non-invasive urodynamics • Non-invasive urodynamics, volunteer recruitment •

Recruitment, epidemiological aspects · Schiedam study • Krimpen study

\begin{abstract}
Introduction: We studied epidemiological aspects of recruitment of volunteers for a non-invasive urodynamic study. Materials and Methods: 9,236 volunteers were invited by 20 general practitioners (GPs), using two different recruitment methods, i.e. by mail only, or during a subsequent visit to the GP's office. Factors influencing the response rates were analyzed. We also tested how much the recruited population of volunteers differed from the general population, by comparing it to another, proven representative study carried out earlier in 1,662 subjects. Results: In the recruited population the prostate volumes were not significantly different from the proven representative study, but the symptom score was statistically significantly higher, although the difference was so small it may be called clinically irrelevant. Recruitment of volunteers in two steps, i.e. asking them first to visit the GP's office, and inviting them there to visit the outpatient clinic, rather than directly inviting them (in writing) to the clinic seemed to lead to a higher response, although this effect could not be statistically discriminated from the difference
\end{abstract}

in response rates between GPs. Conclusion: The population recruited was not urologically different from the general population. The response depended on age, being highest around the age of 60 , and increased with social economic status. It also depended on the GP who recruited the subjects, and/or on the recruitment method.

Copyright $\odot 2006$ S. Karger AG, Basel

\section{Introduction}

With increasing age, the prostate often enlarges, usually as a result of benign prostatic hyperplasia (BPH). In response to the increasing resistance to urinary flow, the urinary bladder function changes. The function of the muscle can be assessed by calculating its contractility from urodynamic measurements of pressure and flow rate during voiding. Presently, such pressure measurements are invasive, i.e. a catheter has to be inserted into the bladder, an expensive and time-consuming procedure, which is uncomfortable for the patient.

We have developed a non-invasive method of measuring the urinary bladder pressure using an external catheter based on an incontinence condom $[1,2]$ and we apply this method in a longitudinal study on healthy males [3].

\section{KARGER}

Fax +4161306 1234

E-Mail karger@karger.ch

www.karger.com (c) 2006 S. Karger AG, Base

0042-1138/06/0772-0159\$23.50/0

Accessible online at: www.karger.com/uin
R. van Mastrigt

Department of Urology, Sector Furore, Room, EE 1630, Erasmus MC PO Box 1738

NL-3000 DR Rotterdam (The Netherlands)

Tel. +31 10408 8058, Fax +31 10408 9451, E-Mail r.vanmastrigt@erasmusmc.nl 


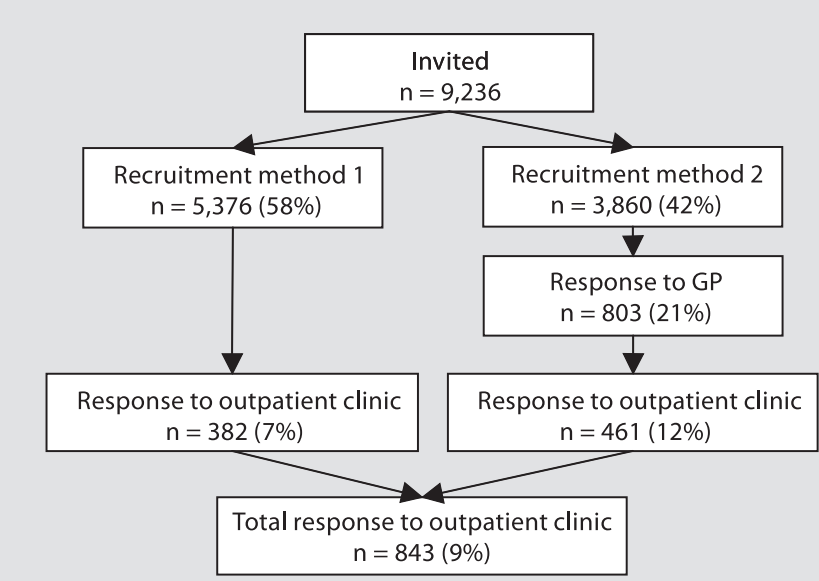

Fig. 1. Flowchart showing the numbers of subjects in each recruitment method of the Schiedam study. The subjects were recruited by two different methods. Recruitment method 1: the volunteers were recruited by letter from their GP, asking them to make an appointment with our outpatient clinic at the Erasmus MC. Recruitment method 2: patients of GPs were asked to visit the GP's practice first for an interview and transabdominal ultrasound examination of the prostate. During this visit they were invited for a complete investigation at the outpatient clinic.

Investigators in most community studies have not considered non-response bias; it is not known whether the study population is truly representative. One preliminary study on potential non-response bias, the Olmsted County Study, suggested that the response might have been affected by concern about urological disease [4]. The age distribution and non-response bias could have a large influence on the measured parameters. We therefore compared our study population with that of another study - the Krimpen study: normal values and determinants of urogenital tract (dys)function in older men [5]. That study is a longitudinal population-based study with 1,688 participants, a response rate of $50 \%$ and a non-responder study, in which the representativeness of the participating population was assessed. The authors described that the participants were representative for the total population: they were similar to those not responding on marital status, educational level, smoking and drinking habits, and treatment of chronic diseases, but had a slightly lower perceived general health status and more LUTS (lower urinary tract symptoms). Adjustment for non-response bias resulted in lower prevalences for all definitions of LUTS used. Additionally, to study possible sources of bias, we tested for differences between responders and non-responders in our study, between responders recruited using two different recruitment methods, and factors influencing the response rates. Using both the comparison with the proven representative study, and the tests for factors influencing the response rates, we address the hypothesis that the population of male volunteers that we recruited for a non-invasive longitudinal urodynamic study is unbiased and represents the general population.

\section{Material and Methods}

\section{Schiedam Study}

Subjects for our longitudinal study were mainly recruited in Schiedam, a municipality near Rotterdam with approximately 75,000 inhabitants. General practitioners (GPs) in Schiedam were approached to invite their male patients. Inclusion criteria were signed informed consent from all volunteers, and ability to void in the standing position with a free flow rate of at least $5 \mathrm{ml} / \mathrm{s}$. Men with a history of any heart condition, known neurological conditions, certain known viral conditions such as being HIV-positive, congenital or acquired conditions or surgery of the lower urinary tract (LUT), current LUT or anticoagulating therapy and diseases altering urinary tract function were excluded. Recruitment took place from November 2001 to December 2003. The study aimed at a rectangular age distribution, i.e. recruiting an equal number of patients in eight cohorts of 5 age years between 38 and 77. Two different recruitment methods were used. Using method 1 the GPs invited the selected men by mail, including a patient information form and a description of the study criteria, and asking them to make an appointment at the outpatient clinic of the Erasmus MC.

Using recruitment method 2 the GPs again primarily applied all the inclusion and exclusion criteria but now invited men by mail to visit the GPs' practices for an interview and transabdominal ultrasound examination of the prostate by the investigator. During this visit they were invited to a complete investigation at the Erasmus MC. During the investigations at the outpatient clinic the prostate volume of each subject was assessed by transabdominal ultrasonography using Aloka ${ }^{\circledR}$ SSD-500 or Aloka ${ }^{\circledR}$ SSD-900 Ultrasound Diagnostic Equipment. The prostate volume was calculated by a two-dimensional prolate spheroid approximation. At sufficient sensation of bladder filling, a free uroflowmetry was done to verify if a maximum flow rate $\left(Q_{\max }\right)$ of at least $5 \mathrm{ml} / \mathrm{s}$ could be achieved. If that condition was satisfied at least one non-invasive bladder pressure measurement was attempted as described before [3]. All subjects completed the International Prostate Symptom Score (IPSS).

In this report, we included all subjects invited by 20 GPs ( $\mathrm{n}=$ 9,236 ), and subdivided these into responders and non-responders. The flowchart (fig. 1) shows the numbers of subjects in each recruitment method. From an official registry a socio-economic continuous rank number (SES), related to all of the almost 4,000 postal areas in The Netherlands, and thus ranging from 1 (high status) to almost 4,000 (low status), was obtained and matched to the subjects by postal code. We stratified the subjects in age groups of 5 years (38-42, 43-47, 48-52, 53-57, 58-62, 63-67, 68-72). 
Krimpen Study

Names and addresses of all registered 3,294 men aged 5075 years were obtained (reference date June 1995) from all general practices of Krimpen aan den IJssel, a commuter suburb near Rotterdam (The Netherlands) with approximately 28,000 inhabitants.

Men who had not undergone radical prostatectomy and had not had prostate or bladder cancer, neurogenic bladder disease or negative advice from their GP, and who were able to complete questionnaires and visit the health center were found eligible. In all cases, the GPs decided whether the patient could enter the study before invitation. The GPs' reasons for excluding any patient were checked by researchers in the electronic medical records. Recruitment took place from August 1995 to January 1998.

Digital rectal examination, transrectal ultrasonography(TRUS), uroflowmetry, post-void residual volume and serum prostate-specific antigen measurements were done at the Urology Outpatient Department.

TRUS was performed with a 7-MHz Brüel \& Kjær multiplane sector scanning probe. The planimetric technique of volume measurement was used. This method involves measuring the surface area of transverse sections taken through the prostate at 5-mm intervals. The average of two intervals multiplied by $5 \mathrm{~mm}$ provides the volume for each step and the cumulative volume allows the total prostatic volume $\left(\mathrm{cm}^{3}\right)$ to be derived. Uroflowmetry was done using a Dantec ${ }^{\circledR}$ Urodyn 1,000 flowmeter. The men were asked to visit the clinic with a full bladder and were instructed not to void in a toilet before that time.

The post-void residual urine volume $(\mathrm{ml})$ in the bladder was computed by transabdominal ultrasonography, using an Aloka machine with a $3.5-\mathrm{MHz}$ handheld probe using the formula $\pi / 6 \times$ $($ width $) \times$ (height $) \times($ depth). The post-void residual was not computed if a man was unable to void in the uroflowmeter. The initial pre-micturitional bladder volume was calculated by summing the voided volume and the residual volume [6].

Descriptives were calculated for the variables measured in both studies. As the two populations differ in age range and in exclusion criteria applied, two subpopulations were formed for comparison. In the restricted Schiedam population only patients with the same age range as in the Krimpen study (50 through 75$)$ were included. The restricted Krimpen population was derived by deleting all subjects with a maximum flow rate $<5 \mathrm{ml} / \mathrm{s}$, as these were excluded in the Schiedam study. Crude differences between the restricted populations were tested for significance using the unpaired t test. The variables IPSS score, $\mathrm{Q}_{\max }$, voided volume and prostate volume were analyzed after logarithmic transformation, because of their positive skewness. Next, these crude differences were corrected for confounding variables, i.e. variables that are observed in both studies (Schiedam and Krimpen), that are known to be differently distributed between the populations and that are also known to have an impact on the four outcome variables considered. The first possibly confounding variable is age. Age is known to have an impact on prostate volume and, due to the design of the present study, its distribution differs between Schiedam (an intentional rectangular distribution) and the population participating in the Krimpen study (a distribution more representative of the general population). The second possibly confounding variable is social economic status. For each outcome variable, the confounding variables age and SES were included in a multiple linear regression model as restricted cubic splines along with population (Schiedam/Krimpen).
The use of restricted cubic splines is a flexible way of efficiently and (almost) optimally adjusting for continuous confounders [7].

Next we analyzed differences in the SES, the age, the recruitment method and the subjects' GPs between responders and nonresponders of the Schiedam study. The subjects were stratified in age groups of 5 years, i.e. 37-42, 43-47, etc. We calculated the mean response rates (i.e. the number of responders divided by the number of invited subjects) for those age groups and GPs. We also categorized the SES in four categories and calculated mean response rates for those four categories. Subsequently, we calculated mean SES and recruitment method for each GP and compared these by analysis of variance. Next we applied multiple logistic regression analysis in order to relate the response simultaneously to these variables. The GP with the lowest response was used as a reference.

Finally, we tested the differences in the distributions of age, SES, IPSS score, $\mathrm{Q}_{\max }$, voided volume and prostate volume between the two groups of responders to the outpatient clinic (Erasmus MC): recruited using methods 1 and 2, using unpaired t tests. Apart from age and SES, all variables were log transformed prior to analysis, as the original variables appeared to have positively skewed distributions. Next these variables were tested simultaneously using multiple logistic regression analysis.

\section{Results}

In the Schiedam study, 9,236 volunteers were invited by 20 GPs. Figure 1 shows that eventually 843 showed up at the outpatient clinic. In the Krimpen study eventually 1,662 men were included. Summary statistics of the two groups of subjects (Schiedam and Krimpen), and the restricted populations that were made comparable in age range and maximum flow rate are presented in table 1 as mean, median, standard deviation and interquartile range.

A simple unpaired t test (i.e. unadjusted) yielded significant differences with $\mathrm{p}<0.0005$ for all variables except age and prostate volume. IPSS, $\mathrm{Q}_{\max }$ and voided volume were higher in Schiedam than in Krimpen. The SES was also higher in Schiedam, indicating a lower social economic status. After adjusting for the covariables age and SES by using restricted cubic splines with 7 knots, the adjusted mean differences in outcome variables between Schiedam and Krimpen remained virtually the same compared to the crude (unadjusted) mean differences.

Table 2 shows the full response rates in the Schiedam study, i.e. the number of subjects presenting at the outpatient clinic (totaling 843), divided by the number invited using both recruitment methods 1 and $2(n=9,236)$. Separate mean response rates are shown for the eight age groups, $20 \mathrm{GPs}$, and four ranges of social economic rank. It appears that the response more or less increased with the age and the SES (a low SES indicates a high social economic status), and was considerably different for different 
Table 1. Descriptives of the covariables age and social economic status and the outcome variables prostate volume, maximum urinary flow rate $\left(\mathrm{Q}_{\max }\right)$, voided volume and International Prostate Symptom Score (IPSS) in both studies (Schiedam, Krimpen)

\begin{tabular}{|c|c|c|c|c|c|c|}
\hline Variables & Study & $\mathrm{n}$ & Mean & Median & SD & IQR \\
\hline \multirow[t]{4}{*}{ Age } & Schiedam & 843 & 55 & 53 & 10 & $48-64$ \\
\hline & -restricted & 538 & 60 & 60 & 8 & $53-67$ \\
\hline & Krimpen & 1,662 & 61 & 60 & 7 & $55-66$ \\
\hline & -restricted & 1,469 & 61 & 60 & 6 & $55-65$ \\
\hline \multirow[t]{4}{*}{ SES ranking $1-4,000$} & Schiedam & 840 & 1,935 & 2,473 & 1,285 & $745-2,889$ \\
\hline & -restricted & 537 & 2,133 & 2,504 & 1,221 & $745-2,889$ \\
\hline & Krimpen & 1,656 & 1,219 & 1,003 & 1,126 & $216-2,851$ \\
\hline & -restricted & 1,466 & 1,213 & 1,003 & 1,124 & $216-2,851$ \\
\hline \multirow[t]{4}{*}{ Prostate volume, $\mathrm{ml}$} & Schiedam & 843 & 36 & 31 & 19 & $24-41$ \\
\hline & -restricted & 538 & 39 & 34 & 22 & $26-47$ \\
\hline & Krimpen & 1,647 & 39 & 35 & 21 & $27-44$ \\
\hline & -restricted & 1,455 & 39 & 35 & 21 & $28-44$ \\
\hline \multirow[t]{4}{*}{$\mathrm{Q}_{\max }, \mathrm{ml} / \mathrm{s}$} & Schiedam & 843 & 18 & 16 & 8 & $11-22$ \\
\hline & -restricted & 538 & 16 & 15 & 7 & $11-20$ \\
\hline & Krimpen & 1,476 & 11 & 10 & 7 & $6-15$ \\
\hline & -restricted & 1,292 & 12 & 11 & 7 & $8-16$ \\
\hline \multirow[t]{4}{*}{ Voided volume, ml } & Schiedam & 843 & 331 & 290 & 186 & $190-444$ \\
\hline & -restricted & 538 & 317 & 282 & 169 & $186-425$ \\
\hline & Krimpen & 1,474 & 181 & 140 & 144 & $80-238$ \\
\hline & -restricted & 1,293 & 198 & 160 & 143 & $95-253$ \\
\hline \multirow[t]{4}{*}{ IPSS } & Schiedam & 843 & 6 & 5 & 5 & $2-8$ \\
\hline & -restricted & 538 & 6 & 5 & 5 & $1-7$ \\
\hline & Krimpen & 1,661 & 5 & 4 & 5 & $1-7$ \\
\hline & -restricted & 1,471 & 5 & 3 & 5 & $1-7$ \\
\hline
\end{tabular}

Results are also given for subpopulations (restricted). In the restricted Schiedam population, only patients with ages 50 through 75 were included. The restricted Krimpen population was derived by deleting all subjects with a maximum flow rate $<5 \mathrm{ml} / \mathrm{s}$.

GPs. In addition to that, it can be seen from figure 1 that the response to the outpatient clinic appeared higher using recruitment method $2(461 / 3,860=0.12)$ than recruitment method $1(382 / 5,376=0.07)$. Table 2 also shows that the mean SES score of the patients invited by the different GPs was considerably different, and that there was a strong relation between the recruitment method used and the GP, caused by the fact that most GPs used only one recruitment method. Only GP 15 and 18 used both methods. Both the SES and the recruitment method depended significantly on the GP according to one-way analysis of variance $(p<0.001)$. When all these variables were entered simultaneously, the multiple logistic regression showed that the significant explanatory variables were GP, age group and SES. No significant interactions were found, and the recruitment method ( 1 or 2 ) was not a significant explanatory variable. The influence of age group on the response was considerable: the odds ratio (OR) of the age group with the highest response (ages 58-62) to that of the age group with the lowest response (ages 38-42) was 2.9. At higher ages the response declined to an OR of 1.7 for the group with ages 73-77 compared to the lowest age group. Similarly, there were large differences between the GPs, the OR varied from 4.7 to 1.2 as compared to the GP with the lowest response (GP 4). 
Table 2. Mean full response in the Schiedam study in the eight age groups of patients from 20 GPs in four social economic classes. In total, 9,236 patients were invited from which 843 made an appointment, and showed up at the outpatient clinic (see fig. 1). Mean response was 0.09

\begin{tabular}{|c|c|c|c|c|}
\hline Age group & Invited, $\mathrm{n}$ & Response & & \\
\hline $38-42$ & 2,081 & 0.05 & & \\
\hline $43-47$ & 1,823 & 0.05 & & \\
\hline $48-52$ & 1,575 & 0.13 & & \\
\hline $53-57$ & 846 & 0.11 & & \\
\hline $58-62$ & 759 & 0.13 & & \\
\hline $63-67$ & 787 & 0.13 & & \\
\hline $68-72$ & 782 & 0.11 & & \\
\hline $73-77$ & 583 & 0.08 & & \\
\hline SES score & Invited, $\mathrm{n}$ & Response & & \\
\hline $91-745$ & 2,823 & 0.12 & & \\
\hline $790-2,504$ & 1,865 & 0.10 & & \\
\hline $2,591-3,474$ & 2,157 & 0.08 & & \\
\hline $3,509-3,874$ & 2,374 & 0.07 & & \\
\hline GP & Invited, $\mathrm{n}$ & Response & SES & $\begin{array}{l}\text { Recruitment } \\
\text { method }\end{array}$ \\
\hline 1 & 469 & 0.06 & 3,028 & 1 \\
\hline 2 & 423 & 0.06 & 2,459 & 1 \\
\hline 3 & 435 & 0.08 & 2,940 & 1 \\
\hline 4 & 418 & 0.04 & 2,656 & 1 \\
\hline 5 & 729 & 0.08 & 2,690 & 1 \\
\hline 6 & 578 & 0.10 & 1,730 & 1 \\
\hline 7 & 645 & 0.07 & 2,401 & 1 \\
\hline 8 & 339 & 0.06 & 3,256 & 1 \\
\hline 9 & 561 & 0.15 & 2,157 & 2 \\
\hline 10 & 442 & 0.10 & 2,497 & 2 \\
\hline 11 & 570 & 0.11 & 2,609 & 2 \\
\hline 12 & 305 & 0.05 & 2,564 & 1 \\
\hline 13 & 428 & 0.11 & 1,177 & 2 \\
\hline 14 & 684 & 0.06 & 2,480 & 1 \\
\hline 15 & 233 & 0.12 & 1,531 & 1.82 \\
\hline 16 & 384 & 0.13 & 1,242 & 2 \\
\hline 17 & 1,147 & 0.11 & 868 & 2 \\
\hline 18 & 208 & 0.19 & 2,558 & 1.66 \\
\hline 19 & 130 & 0.12 & 3,124 & 1 \\
\hline 20 & 105 & 0.09 & 2,382 & 1 \\
\hline
\end{tabular}

The mean SES and mean recruitment method are also shown for each GP. GPs 15 and 18 used both recruitment methods. For them the (two digits after the decimal point of the) displayed number reflect the percentage of patients recruited using method 2 .
According to the t-test (table 3 ) the IPSS score and voided volume were not significantly different between the two groups recruited differently ( $\mathrm{p}$ values 0.51 and 0.94 respectively), but all other variables were. Group 1 (the group invited by mail to make an appointment at the outpatient clinic) was on average older than group 2 (invited to visit the GP office first), had a lower social economic status (a higher SES), a lower flow rate, and a lower prostate volume. In the multiple logistic regression analysis, IPSS was again not significant $(\mathrm{p}=0.89)$ and age became only marginally significant $(\mathrm{p}=0.07)$, given the other highly significant variables $(p<0.001)$ : SES, $Q_{\max }$, voided volume and prostate volume. It appeared that voided volume became significant when adjusted for the other variables (especially $\mathrm{Q}_{\max }$ ) in the model. If voided volume increased by $10 \%$ (given the other variables in the model) then the odds of belonging to group 2 was multiplied by 0.95 (95\% CI: $0.92-0.98$ ). If $\mathrm{Q}_{\max }$ increased by $10 \%$ then the odds of belonging to group 2 was multiplied by 1.10 (95\% CI: 1.05-1.14). If prostate volume increased by $10 \%$ then the odds of belonging to group 2 was multiplied by 1.06 (95\% CI: 1.02-1.10). The effect of the uniform socio-economic ranking (ranging from 1 to 4,000) was significantly modified by age and was given by an OR per 1,000 points higher SES of around 1.3 in the lowest age group to 0.9 in the highest age groups.

\section{Discussion}

We studied epidemiological aspects of a population of 843 volunteers recruited for a longitudinal non-invasive study of changes in urinary bladder contractility secondary to $\mathrm{BPH}$ performed in Schiedam. Our aim was to establish if the population might be biased, or if it may be considered a representation of the general population. To this end we compared a number of variables with a proven representative study, performed in Krimpen. We also studied differences between responders and non-responders, differences between responders recruited using two different methods, and factors influencing the response rates in our population, to assess possible sources of bias.

Global inspection of both (restricted) populations in table 1 seems to show that the SES of the Schiedam population was higher than that of the Krimpen population, which indicates a lower social economic status. Prostate volumes were not significantly different, and although symptom scores were significantly different, the differences were so small they may be called clinically insignificant 
Table 3. T-test of age, SES, IPSS score, maximum flow rate, voided volume and prostate volume between the two groups of responders to the outpatient clinic (Erasmus MC) recruited using methods 1 and 2 in the Schiedam study

\begin{tabular}{lllccc}
\hline Explanatory variables & $\begin{array}{l}\text { Recruitment } \\
\text { method }\end{array}$ & $\mathrm{n}$ & Mean & $\mathrm{SD}$ & p value \\
\hline Age & 1 & 382 & 56 & 9.9 & $<0.001$ \\
& 2 & 461 & 54 & 11 & \\
SES & 1 & 381 & 2,335 & 1,180 & $<0.001$ \\
& 2 & 459 & 1,602 & 1,275 & \\
IPSS $^{\mathrm{a}}$ & 1 & 382 & 6.14 & 4.9 & 0.51 \\
\multirow{2}{*}{ Maximum flow rate $^{\mathrm{a}}, \mathrm{ml} / \mathrm{s}$} & 2 & 461 & 5.60 & 4.4 & \\
\multirow{2}{*}{ Voided volume $^{\mathrm{a}}, \mathrm{ml}$} & 2 & 382 & 16 & 7 & $<0.001$ \\
& 1 & 461 & 19 & 9 & \\
Prostate volume $^{\mathrm{a}}, \mathrm{ml}$ & 2 & 382 & 322 & 166 & 0.94 \\
& 1 & 461 & 339 & 201 & \\
& 2 & 382 & 35 & 22 & 0.04 \\
\end{tabular}

a The variables IPSS score, maximum flow rate, voided volume and prostate volume were tested after logarithmic transformation. The descriptives displayed however are from the untransformed variables. (the IPSS ranges from 0 to 35). Prostate volumes were measured using different methods in both studies but these methods were compared in a separate study, and were found not to cause significant differences [8].

Maximum flow rate and voided volume both seemed higher in the Schiedam population. This is at least partly due to the different measurement technique applied. While the subjects in the Krimpen study were asked to void in a flow meter if they could, the Schiedam subjects were asked if they had a full bladder, and were encouraged to delay voiding and drink water until this was the case. As a consequence, the Schiedam subjects voided higher volumes. There is a positive relation between flow rate and voided volume: at increasing voided volume, maximum flow rates increase. Therefore, the higher voided volumes in Schiedam may (partly) explain the higher flow rates. It can therefore be concluded that the restricted population from the Schiedam study (i.e. only the subjects with an age range of 50-75) was urologically not different from the population tested in Krimpen, which has been shown to represent the general population [5], although the social economic status was significantly higher in Krimpen.

In the literature, three main types of bias have been distinguished: selection bias, information bias and confounding bias [9]. Confounding, in the context of the ongoing longitudinal study, would mean that the effect (a change in urinary bladder contractility) is caused by other factors than prostatic enlargement. The most likely source of such confounding is aging, especially in view of the knowledge that in a cross-sectional study, bladder contractility in females significantly decreased with age while in males this was not the case (in males it was probably masked by compensation) [10]. Although this possible source of bias has to be considered when analyzing the outcomes of the longitudinal study, it is not caused by the differences between the study population and the general population, and therefore will not be considered here. In the context of the longitudinal study, information bias would take the form of regression to the mean, i.e. extreme values in the first evaluation will tend to be closer to the center of the distribution in a later measurement [11]. Such regression could affect the longitudinal study, and is one of the reasons for comparing the recruited population with the Krimpen population. Selection bias is the most likely bias in the study. The way in which subjects were recruited influences the composition of the study population, and thus may influence its outcome. Apart from the intentionally rectangular age distribution, which is different from the age distribution in the general population, the study might for instance be biased by self-selection, i.e. perhaps subjects with more-than-average symptoms did more frequently respond. Additionally, subjects with serious symptoms, such as a very low flow rate, were excluded. For this reason we studied factors influencing the response rate of the subjects. We found that the response depended on age, SES and the GP who invited the subjects. When tested separately, also the recruitment method used (i.e. by direct mail from the GP, asking his patient to make an 
appointment with the outpatient clinic, or by inviting the patients to the GP office first for an interview and transabdominal ultrasound examination of the prostate during this visit they were invited for a complete investigation at the clinic) made a significant difference. However, when all these variables were entered simultaneously in a multiple logistic regression analysis, the recruitment method was not a significant explanatory variable anymore. This was caused by the fact that most GPs only used one of the two recruitment methods, so that there was a strong relation between GP and recruitment method as explanatory variables. The same effect was seen for GP and social economic status. The response was highest at the age of 65 and it increased with social economic status.

The distribution of the IPSS score was about the same in both groups of responders to the outpatient clinic (Erasmus MC): recruited using methods 1 and 2.

All other variables studied were significantly different: voided volume, SES and age were higher in group 1 compared to group 2 (although age was only marginally significant); maximum flow rate $\left(\mathrm{Q}_{\max }\right)$ and prostate volume were lower. However, as judged from the means, the differences in age, voided volume and prostate volume were small, and also the ORs of the logistic regression analyses were limited. It thus appears that although both groups were different, the difference was very limited and may be called clinically irrelevant.

\section{Conclusion}

In general we conclude that several factors influenced the response rate of subjects we recruited for a non-invasive longitudinal study of changes in bladder contractility secondary to bladder outlet obstruction. Age and social economic status did, the response being highest around the age of 60 years and lower at lower and higher ages, and increasing with social economic status. Recruitment of volunteers in two steps, i.e. asking them first to visit the GP's office, and inviting them there to visit the outpatient clinic, rather than directly inviting them (in writing) to the clinic seemed to lead to a higher response, although this effect could not be statistically discriminated from the difference in response rates between GPs. This latter difference might be ascribed to the relation between subject and his GP, although also a difference of mean social economic status between patient populations of different GPs may have influenced that. In spite of these dependencies, we found that when the recruited population was corrected for intentional differences in age distribution and methodological differences in flow rates, it was not urologically different from the general population.

\section{Acknowledgements}

This study was supported by the Dutch Kidney Foundation (PC85), and the foundations 'Vereniging Trustfonds Erasmus Universiteit', 'Stichting Bevordering Volkskracht' and 'Stichting Aelwijn Florisz.' It was approved by the Medical Ethics Committee of the Erasmus Medical Centre, No. 202.680/2001/148.

\section{References}

$>1$ Pel JJM, van Mastrigt R: Non-invasive measurement of bladder pressure using an external catheter. Neurourol Urodyn 1999; 18:455469. Authors' reply: Neurourol Urodyn 1999; 18:472-475.

$>2$ Pel JJM, van Mastrigt R: The variable outflow resistance catheter: a new method to measure bladder pressure noninvasively. J Urol 2001; 165:647-652.

$>3$ Huang Foen Chung JWNC, Bohnen AM, Pel JJM, Bosch JLHR, Niesing R, van Mastrigt R: Applicability and reproducibility of the condom catheter method for measuring the isovolumetric bladder pressure. Urology 2004; 63:56-60.
4 Panser LA, Chute CG, Guess HA, et al: The natural history of prostatism: the effects of nonresponse bias. Int J Epidemiol 1994;23: 1198-1205.

5 Blanker MH, Groeneveld FP, Prins A, Bernsen RM, Bohnen AM, Bosch JL: Strong effects of definition and nonresponse bias on prevalence rates of clinical benign prostatic hyperplasia: the Krimpen study of male urogenital tract problems and general health status. BJU Int 2000;85:665-671.

6 Blanker MH: Normal values and determinants of urogenital tract (dys)function in older men: the Krimpen Study; thesis, Erasmus MC, 2002.

7 Harrell FE Jr: Regression Modeling Strategies. New York, Springer, 2001, pp 20-24.
-8 Huang Foen Chung JWNC, Raaijmakers R, de Vries SH, Postma R, Bosch JLHR, van Mastrigt R: Prostate volume ultrasonography: the influence of transabdominal versus transrectal approach, device type and operator. Eur Urol 2004; 46:352-356.

9 Kleinbaum DG, Kupper LL, Morgenstern H Epidemiologic Research. Belmont/CA, Lifetime Learning Publications, 1982.

10 Van Mastrigt R: Age dependence of urinary bladder contractility. Neurourol Urodyn 1992; 11:315-317.

11 Davis CE: The effect of regression to the mean in epidemiologic and clinical studies. Am J Epidemiol 1976;104:493-498. 